\title{
A comparative study on the efficacy of first vs second blood culture set in the diagnosis of bacteraemia in Central Referral Hospital, Sikkim, India
}

\author{
Karma G. Dolma ${ }^{1}$, Deepan Gautam ${ }^{2}$, Kh. Pooja Devi ${ }^{3}$, T. S. K. Singh ${ }^{4}$ \\ ${ }^{1}$ Dr. Karma G. Dolma, Assistant Professor, Department of Microbiology, Sikkim Manipal Institute of Medical Sciences, \\ ${ }^{2}$ Mr. Deepan Gautam, Tutor, Department of Microbiology, Sikkim Manipal Institute of Medical Sciences, ${ }^{3}$ Ms. Kh. \\ Pooja Devi, Msc (Medical Microbiology), Department of Microbiology, Sikkim Manipal Institute of Medical Sciences, \\ ${ }^{4}$ Dr (Prof) T. S. K. Singh, Head of Department, Department of Microbiology, Sikkim Manipal Institute of Medical \\ Sciences, Gangtok, Sikkim, India.
}

Address for correspondence: Dr. Karma G. Dolma, Assistant Professor, Department of Microbiology, Sikkim Manipal Institute of Medical Sciences (SMIMS) and Central Referral Hospital (CRH), $5^{\text {th }}$ Mile Tadong, Gangtok, Sikkim, India - 737 102. Ph. No.+91-3592-205615 Fax : (Office) (03592) 231496; Mobile: (91) 9434025537. Email: gyur_skyscraper@hotmail.com

\begin{abstract}
Background: An important but controversial subject is the number of blood culture sets required for the diagnosis of blood stream infection (BSI) and also the use of appropriate antibiotics to treat bacteremia. This paper focuses on the need of two blood culture set in comparison to one blood culture in the diagnosis of bacteraemia. Methods: First and second sets were collected aseptically from two different sites at an interval of about one hour from all clinically suspect patients of bacteremia. The samples were processed in Bact/ALERT3D system and further identified in VITEK 2 compact. Results: Second blood culture set yielded higher rates of positive cultures (63\%) than first set (37\%). The common bacterial isolates were Coagulase negative Staphylococcus (CoNS) 29 (28\%), followed by Staphylococcus aureus 20 (20\%), Escherichia coli $13(13 \%)$ and Klebsiella pneumonia 11(11\%). Methicillin resistance was observed in $90 \%$ of $S$. aureus isolates. All Gram positive bacteria were found sensitive to vancomycin. In Gram-negative organisms, extended spectrum $\beta$-lactamases (ESBL) was observed in $40.5 \%$ isolates and resistance to carbapenems was found to be $37.8 \%$. Discussion/Conclusion: In India, most hospitals routinely use single aerobic blood culture. The isolation of CoNS in blood is difficult to interpret hence, proper collection, processing, and relevant clinical information can significantly reduced the chances of contamination. Automated blood culture system can significantly shorten the length of time for isolation and identification compared to the manual techniques which takes about seven days. Resistance to antibiotics is a matter of concern that can result in ineffective treatment.
\end{abstract}

Keywords: Antibiotic susceptibility test, Bacteraemia, Bact/ALERT3D system, First and second blood culture, Resistance.

\section{Introduction}

Bacterial pathogens isolated from BSI are a leading cause of significant patient morbidity and mortality. It has been estimated that approximately 200,000 cases of bacteremia occur worldwide with mortality rates ranging from 20 to $50 \%$ [1]. In a way, BSI increases the mortality rate, prolongs patient's stay in the hospital and

Manuscript received $5^{\text {th }}$ June 2016

Reviewed: 14 ${ }^{\text {th }}$ June 2016

Author Corrected: 24 ${ }^{\text {th }}$ June 2016

Accepted for Publication $5^{\text {th }}$ July 2016 leads to increased health care costs [2,3]. The blood culture is the gold standard technique for the diagnosis of bacteraemia. It not only has a great diagnostic and prognostic significance but also provides essential information for the evaluation of a variety of diseases [4]. Blood culture is a common laboratory investigation where traditionally, blood is inoculated into culture medium and sub-cultured for positive growth however drawback of manual culture techniques mainly includes 
longer duration for isolation and identification of organisms, increases more false positives cultures from contaminated blood samples and decreased sensitivity for fastidious pathogens $[5,6]$. It is seen that advanced method like automated culture system (Bact/ALERT3D system) is the answer to resolve the following problems. Apart from that, the isolation of clinically significant microorganisms from a case of bacteraemia indicates either failure of host defence to contain an infection at its primary site or that the clinicians have failed to adequately treat the infection [7].

An important issue is the number of blood culture sets required for the diagnosis of BSI and reporting of the organism as well as their antimicrobial profile on time.

The optimal number of blood cultures that should be obtained in a febrile patient varies according to the suspected diagnosis or clinical condition, the suspicion of underlying infection, and the urgency of the need for treatment [8-10]. Blood culture remains one of the most important microbiological tests available to the healthcare team; hence people who perform this test must have a sound knowledge of this important diagnostic tool. Keeping this in mind, this study was designed to compare the policy of first blood culture set over second blood culture set in the diagnosis and treatment of bacteraemia.

\section{Materials and Methods}

Study design: Prospective cross-sectional study.

Setting: Department of Microbiology, Sikkim Manipal Institute of Medical Sciences (SMIMS), Sikkim.

Study period: October 2014 - September 2015(1 year).

\section{Results}

In the present study, out of the total 150 samples received, $82(55 \%)$ were from males and $68(45 \%)$ were from females. Maximum number of the samples were obtained from patients within the age group 21-40 years 52 (35\%) which was closely followed by 48(32\%) of the age group 41-60 years (Table 1).

It was also observed that age was not statistically significant with the number of blood cultures set $(p=0.7010)$.

The samples were obtained from various departments; majority of them were from the medicine department $92(61.4 \%)$; followed by emergency $28(18.6 \%), 16(10.6 \%)$ of them were from the surgery department, $8(5.4 \%)$ from the dialysis and only $6(4 \%)$ from the OBG department. It was observed that samples from various department in correlation to the positive blood culture was statistically significant $(\mathrm{p}<0.001)$ (Table 2$)$.

In this study, the primary infection commonly diagnosed was pyrexia of unknown origin (PUO) 49 (32.6\%) followed by genitourinary infection $29(19.4 \%)$. It was also observed that primary infection was found to be statistically insignificant with the number of blood culture in the study $(p=0.5347)$ (Table 3$)$.
Study population: All clinically diagnosed cases of bacteremia attending Central Referral Hospital (CRH), Sikkim.

Sample collection: Blood samples were collected from patients in the general ward and critical care units by nursing staff or by trained phlebotomist before the administration of antimicrobial drugs.

Before collecting the blood sample, the skin was disinfected with $70 \%$ alcohol and $2 \%$ tincture of iodine the first blood culture set, about $5-10 \mathrm{ml}$ of adult blood, $2-3 \mathrm{ml}$ of children and $1-2 \mathrm{ml}$ of infant blood was collected from peripheral vein and the second set was collected after an interval of about one hour from a different site aseptically and immediately transported to the laboratory for further processing. In the Bact/ALERT 3D system (bioMerieux) incubation of the bottles were continued for seven days until microbial growth was detected.

Gram staining was performed from the positive blood culture and subculture on blood agar, chocolate and MacConkey agar and incubated at $37^{\circ} \mathrm{C} \pm 2^{\circ} \mathrm{C}$. The positive growth was further processed for identification and sensitivity on VITEK 2 Compact (bioMerieux, France). VITEK 2 Compact is an automated microbial identification and antibiotic susceptibility testing system. It also demonstrates the Minimum Inhibitory Concentration levels of bacteria on the commonly used antibiotics in clinical practice. The VITEK 2 Compact system interpretation of the antibiotic is based on the Clinical Laboratory Standards Institute (CLSI) guidelines. 
Table 1: Positive blood culture and age distribution of bacteraemia suspected patients at Central Referral Hospital from October 2014 - September 2015.

\begin{tabular}{|c|c|}
\hline Age-group $(\mathbf{n = 1 5 0})$ & Positive blood culture $\mathbf{n = 1 0 2}(\mathbf{\%})$ \\
\hline$\leq 20(12)$ & $9(75 \%)$ \\
\hline $21-40(52)$ & $32(61.5 \%)$ \\
\hline $41-60(48)$ & $35(72.9 \%)$ \\
\hline $61-80(28)$ & $18(64.2 \%)$ \\
\hline$\geq 80(10)$ & $8(80 \%)$ \\
\hline
\end{tabular}

Table 2: Positive blood culture and department distribution of bacteraemia suspected patients at Central Referral Hospital from October 2014 - September 2015.

\begin{tabular}{|c|c|}
\hline Department $(\mathbf{n}=\mathbf{1 5 0})$ & Positive blood culture n=102(\%) \\
\hline Medicine (92) & $84(91.3 \%)$ \\
\hline Emergency (28) & $10(35.7 \%)$ \\
\hline Surgery (16) & $04(25 \%)$ \\
\hline Dialysis (08) & $02(25 \%)$ \\
\hline OBG (06) & $02(33.3 \%)$ \\
\hline
\end{tabular}

Table 3: Positive blood culture and primary infection of bacteraemia suspected patients at Central Referral Hospital from October 2014 - September 2015.

\begin{tabular}{|c|c|}
\hline Primary source of Infection $(\mathbf{n = 1 5 0 )}$ & Positive blood culture n=102(\%) \\
\hline PUO (49) & $36(73.4 \%)$ \\
\hline Genitourinary Infection (29) & $19(65.5 \%)$ \\
\hline Surgical site Infection (22) & $16(72.7 \%)$ \\
\hline Cardiovascular sites (19) & $09(47.3 \%)$ \\
\hline Respiratory Tract Infection (17) & $13(76.4 \%)$ \\
\hline Gastrointestinal tract Infection (14) & $09(64.2 \%)$ \\
\hline
\end{tabular}

Table 4: List of Gram negative and Gram positive organism isolated from first and second blood culture set

\begin{tabular}{|c|c|c|}
\hline Organisms & First blood culture set $(\mathbf{n}=38)$ & Second blood culture set $(\mathbf{n}=64)$ \\
\hline Gram negative bacilli & & $09(14 \%)$ \\
\hline Escherichia coli & $04(10.5 \%)$ & $07(11 \%)$ \\
\hline Klebsiella pneumoniae & $04(10.5 \%)$ & $04(6 \%)$ \\
\hline Pseudomonas spp & $03(7.8 \%)$ & $04(6 \%)$ \\
\hline S. paratyphi A & $02(5 \%)$ & $02(3 \%)$ \\
\hline Acinetobacter spp. & - & $02(3 \%)$ \\
\hline Enterobacter cloacae & - & $01(1.5 \%)$ \\
\hline Alcaligens faecalis & - & $01(1.5 \%)$ \\
\hline Stenotrophomonas maltophilia & - & $15(23 \%)$ \\
\hline Gram positive cocci & $05(13 \%)$ & $06(9 \%)$ \\
\hline Staphylococcus aureus & $16(42 \%)$ & $13(20 \%)$ \\
\hline Enterococcus spp. & $04(10.5 \%)$ & \\
\hline CoNS & & \\
\hline
\end{tabular}


Table 5: Resistance pattern of Gram positive bacteria isolated from bacteraemia suspected patients in Central Referral Hospital from October 2014 - September 2015

\begin{tabular}{|c|c|c|c|}
\hline Antibiotics & $\begin{array}{c}\text { CoNS } \\
\mathbf{n}(\%)\end{array}$ & $\begin{array}{c}\text { Staphylococcus aureus } \\
\mathbf{n}(\%)\end{array}$ & $\begin{array}{c}\text { Enterococcus spp. } \\
\mathbf{n}(\%)\end{array}$ \\
\hline Oxacillin & $25(86 \%)$ & $18(90 \%)$ & $5(50 \%)$ \\
\hline Cefoxitin & $10(34 \%)$ & $7(35 \%)$ & $0(0 \%)$ \\
\hline Cotrimoxazole & $6(21 \%)$ & $11(55 \%)$ & $5(50 \%)$ \\
\hline Gentamicin & $12(41 \%)$ & $6(30 \%)$ & $0(0 \%)$ \\
\hline Tetracycline & $10(34 \%)$ & $5(25 \%)$ & $0(0 \%)$ \\
\hline Ciprofloxacin & $18(62 \%)$ & $6(30 \%)$ & $4(40 \%)$ \\
\hline Clindamycin & $10(34 \%)$ & $6(30 \%)$ & $0(0 \%)$ \\
\hline Linezolid & $6(21 \%)$ & $0(0 \%)$ & $0(0 \%)$ \\
\hline Vancomycin & $0(0 \%)$ & $0(0 \%)$ & 0 \\
\hline
\end{tabular}

Table 6: Resistance pattern of Gram negative bacteria isolated from bacteraemia suspected patients in Central Referral Hospital from October 2014 - September 2015

\begin{tabular}{|c|c|c|c|c|}
\hline Antibiotics & $\begin{array}{c}\text { Escherichia coli } \\
\mathbf{n}(\%)\end{array}$ & $\begin{array}{c}\text { Klebsiella pneumoniae } \\
\mathbf{n}(\%)\end{array}$ & $\begin{array}{c}\text { Pseudomonas spp } \\
\mathbf{n}(\%)\end{array}$ & $\begin{array}{c}\text { S. paratyphi A } \\
\mathbf{n}(\%)\end{array}$ \\
\hline Ampicillin & $10(77 \%)$ & $6(55 \%)$ & $2(29 \%)$ & $4(67 \%)$ \\
\hline Ceftazidime & $6(46 \%)$ & $4(36 \%)$ & $2(29 \%)$ & $3(50 \%)$ \\
\hline Cefepime & $7(54 \%)$ & $6(55 \%)$ & $2(29 \%)$ & $3(50 \%)$ \\
\hline Ceftriaxone & $7(54 \%)$ & $3(27 \%)$ & $2(29 \%)$ & $1(17 \%)$ \\
\hline Imipenem & $5(38 \%)$ & $7(64 \%)$ & $3(43 \%)$ & $2(33 \%)$ \\
\hline Meropenem & $2(15 \%)$ & $4(36 \%)$ & $3(43 \%)$ & $2(33 \%)$ \\
\hline Gentamicin & $4(31 \%)$ & $3(27 \%)$ & $2(29 \%)$ & $1(17 \%)$ \\
\hline Amikacin & $2(15 \%)$ & $2(18 \%)$ & $2(29 \%)$ & $1(17 \%)$ \\
\hline Tetracycline & $4(31 \%)$ & $4(36 \%)$ & - & $1(17 \%)$ \\
\hline Nitrofurantoin & $2(15 \%)$ & $3(27 \%)$ & - \\
\hline
\end{tabular}

Out of 150 samples, blood cultures were positive in $102(68 \%)$ samples and negative in $48(32 \%)$ samples. In the present study, second blood culture set was observed to have higher yield rates than first blood culture set. Second blood culture set were $64(63 \%)$ and first blood culture set were 38 (37\%). It was observed that microorganism like Acinetobacter spp., Enterobacter cloacae, Alcaligens faecalis and Stenotrophomonas maltophilia were isolated from second blood culture set and the first blood cultures were negative. Out of the 64 second blood culture positives, Gram negative bacilli were 30 (47\%) and Gram-positive cocci (GPC) were 34 (53\%) whereas, in 38 first blood culture, GNB were 13 (34\%) and GPC in $25(66 \%)$. We have not found any mixed infections in our study. The predominant bacteria isolated CONS 29 (28\%), followed by S. aureus 20 (20\%) and E. coli 13 (13\%) and K. pneumoniae 11(11\%).

In second blood culture set, among the GNB, E. coli is the most common 9 (14\%) followed by K. pneumonia 7 (11\%), Pseudomonas spp and S. paratyphi A 4 (6\%) each, Acinetobacter spp. and Enterobacter cloacae 2 (3\%) each. Among the Gram positive cocci, Staphylococcus aureus 15 (23\%) was the most isolated organism followed by CONS 13 (20\%) and Enterococcus spp. 6 (9\%). In first blood culture set, E. coli was the most common 4 (10.5\%) followed by K. pneumoniae $4(10.5 \%)$, Pseudomonas spp $3(7.8 \%)$ and S. paratyphi A $2(5 \%)$. In the Gram positive cocci, CONS 16 (42\%) and S. aureus 5(13\%) was the most isolated organism followed by Enterococcus spp. 4 (10.5\%) (Table 4). Sixty seven samples $(66 \%)$ were found to be positive within the first $24 \mathrm{hrs}$ of incubation, followed by 25 samples (25\%) between $24-48 \mathrm{hrs}$ of incubation of blood samples and the remaining bottles were positive after $48 \mathrm{hrs}$ of incubation. 
The antibiotic susceptibility pattern showed that all Gram positive bacteria were $100 \%$ sensitive to vancomycin. CoNS showed $86 \%$ resistance to oxacillin and $62 \%$ to ciprofloxacin, while resistance was much lower in case of linezolid and cotrimoxazole (21\%) each; clindamycin, tetracycline and cefoxitin (34\%) each. S. aureus was highly sensitive to vancomycin and linezolid (100\%); however $90 \%$ of S. aureus isolates were found to be MRSA. Enterococcus spp. was also found to be $100 \%$ sensitive to cefoxitin, cotrimoxazole, tetracycline, ciprofloxacin, linezolid and vancomycin. Antimicrobial resistance levels for the Gram-negative organisms, causing blood stream infections were found to be varied. ESBL was observed in $40.5 \%$ and carbapenem resistance was observed in $37.8 \%$ of Gram negative isolates. (Table 5 \& 6).

\section{Discussion}

The use of single blood culture in a developing country like India is a common practice where several studies have addressed the issue of the number of blood cultures needed to detect bacteraemia [9, 11-12]. Use of more than one blood culture generally increases the sensitivity of isolation of organism. Clinical and Laboratory Standards Institute (CLSI) recommends four $10 \mathrm{ml}$ bottles to detect $90-95 \%$ of bacteraemias [13] Current recommendations include collecting at least two sets of, each $20 \mathrm{ml}$ of blood distributed equally between an aerobic and an anaerobic bottle from two distinct sites [7, 14]. Lee et al (2007) reported that in order to detect $90 \%$ of true bacteraemia, 2 blood culture sets should be taken in a 24 hour period, however to detect $>99 \%$ up to 4 blood culture sets maybe necessary [8]. However, it is seen that generally most hospitals prefer the use of single aerobic blood culture, taking into account the high cost for culture, time factor, labor intensity, patient's refusal to draw out blood and the need for quick result. In the present study, second blood culture set 64 (63\%) was observed to have higher yield rates than the first set 38 (37\%). Similar observation was seen in a study from a super specialty hospital in New Delhi where paired blood cultures were $13.70 \%$ positive and single blood culture was $4.59 \%$ positive [15]. The use of 2 aerobic blood cultures has also been controversial as many studies have recommended the pairing of anaerobic blood culture with aerobic blood culture and many others have contradicted the use of anaerobic-aerobic blood culture [16-20]. However the result of our study supports the use of two aerobic blood cultures.

In our study, $42 \%$ of infections were caused by Grampositive and $58 \%$ by Gram-negative bacteria. Out of the 64 second blood culture set, Gram negative bacilli were 30 (47\%), Gram-positive cocci were 34(53\%). Similarly in 38 first blood set, GNB were 13 (34\%), GPC in 25 $(66 \%)$. Several studies have shown marginally higher prevalence of Gram-positive and lower prevalence of Gram negative organisms [21-24] and on the contrary, Gram-negative bacteria have been reported as the commonest cause of bacteremia in hospitalized febrile patients in many developing countries [25-27]. The Bact/Alert system isolated many common organisms like CoNS, E. coli, Klebsiella spp, S. aureus but other rare organism like Stenotrophomonas spp. was also isolated. It is known that microorganism like S. aureus, E. coli and other Enterobacteriaceae, P. aeruginosa, S. pneumoniae represent true infection [7]. In our study CoNS were the most commonly isolated bacteria 29 $(28 \%)$ as so have many other studies [22, 23, 28-30].

On the whole, CoNS are difficult to interpret, because in only $12 \%-15 \%$ of the blood isolates were found to be the causative agent in PUO [31]. It is the physicians who ultimately make the final judgment, taking into account not only the laboratory findings but also the clinical condition of the patient. Therefore, clinical information and more than one blood culture can considerably aid to decide whether an isolate is more likely to be significant pathogen or a contaminant. In the present study, most of the isolates $(66 \%)$ were recovered within $24 \mathrm{hrs}$ and $25 \%$ within $48 \mathrm{hrs}$ due to availability of automated blood culture system. Similarly, Tarai et al (2012) were able to recover most of the isolates $(95.8 \%)$ within $48 \mathrm{hr}$ from the automated blood culture system. This system supports the recovery of most of the organisms and VITEK 2 compact also identifies diverse group of species faster [15]. The use of blood culture systems still remain the gold standard for the detection of bacteremia and septicemia. It is important to understand the process from collection to obtaining a result to assist in the interpretation and improve the clinical outcome at the earliest. Automated blood culture and identification methods has significantly reduced the time required for processing of samples and has also facilitated the yield of various organisms including rare organisms.

The study also assessed the antimicrobial susceptibility pattern among the key pathogens causing bloodstream infections to the commonly used antibiotics in the hospital. All Gram positive bacteria were $100 \%$ 
sensitive to vancomycin but were found to be MRSA (90\%). This is of concern as all penicillin groups of drugs are often used for initial and empirical treatment of Staphylococcal infections. In gram negative organisms, $40.5 \%$ of ESBL was observed to commonly used antibiotics; it is a matter of concern as this could further complicate management and increase morbidity and mortality.

\section{Conclusion}

There is a need to use more than a one blood culture for earlier detection of bacteraemia as there is a higher chance of isolation of organism. Also the use of automated blood cultures; currently represent the "gold standard" for diagnosis of bacteraemia. However, the process of proper blood collection, proper isolation and identification process and the pertinent clinical information will help to obtain the correct result and improve the clinical outcome at the earliest.

Funding: Nil, Conflict of interest: None initiated, Permission from IRB: Yes

\section{Reference}

1. Forbes BA, Sahm DF, Weissfeld AS. Bailey and Scott's Diagnostic Microbiology. $12^{\text {th }}$ edition. St. Louis, Missouri: Mosby Elsevier; 2007.

2. Tziamabos AO, Kasper DL. Principle and practice of infectious diseases. Frank Polizano J 2005, 26:2810-16.

3. Madsen KM, Schønheyder HC, Kristensen B, Sørensen HT. Secular trends in incidence and mortality of bacteraemia in a Danish county 1981-1994. APMIS. 1999 Mar;107(3):346-52.

4. Murty DS, Gyaneshwari M. Blood cultures in paediatric patients: a study of clinical impact. Indian $\mathrm{J}$ Med Microbiol. 2007 Jul;25(3):220-4.

5. Gaibani P, Rossini G, Ambretti S, Gelsomino F, Pierro AM, Varani S, Paolucci M, Landini MP, Sambri V. Blood culture systems: rapid detection--how and why? Int J Antimicrob Agents. 2009;34 Suppl 4:S13-5. doi: 10.1016/S0924-8579(09)70559-X.

6. Mancini N, Carletti S, Ghidoli N, Cichero P, Burioni $\mathrm{R}$, Clementi M. The era of molecular and other nonculture-based methods in diagnosis of sepsis. Clin Microbiol Rev. 2010 Jan;23(1):235-51. doi: 10.1128 / CMR.00043-09.
7. Weinstein MP. Current blood culture methods and systems: clinical concepts, technology, and interpretation of results. Clin Infect Dis. 1996 Jul; 23(1):40-6.

8. Lee A, Mirrett S, Reller LB, Weinstein MP. Detection of bloodstream infections in adults: how many blood cultures are needed? J Clin Microbiol. 2007 Nov;45(11):3546-8. Epub 2007 Sep 19.

9. Washington JA 2nd. Blood cultures: principles and techniques. Mayo Clin Proc. 1975 Feb;50(2):91-8.

10. Szymczak EG, Barr JT, Durbin WA, Goldmann DA. Evaluation of blood culture procedures in a pediatric hospital. J Clin Microbiol. 1979 Jan;9(1): 88-92.

11. Weinstein MP, Reller LB, Murphy JR, Lichtenstein KA. The clinical significance of positive blood cultures: a comprehensive analysis of 500 episodes of bacteremia and fungemia in adults. I. Laboratory and epidemiologic observations. Rev Infect Dis. 1983 Jan-Feb;5(1): 35-53.

12. Weinstein MP, Joho KL, Quartey SM. Assessment of the third blood culture: does it increase detection of bacteremia [abstract no C150]. In: Program and abstracts of the 94th General Meeting of the American Society for Microbiology (Las Vegas). Washington, DC: American Society for Microbiology, 1994:517.

13. Clinical and Laboratory Standards Institute (CLSI). Principles and Procedures for Blood Cultures; Approved Guideline. CLSI document M47-A. Wayne, PA: Clinical and Laboratory Standards Institute 2007.

14. Cockerill FR 3rd, Wilson JW, Vetter EA, Goodman KM, Torgerson CA, Harmsen WS, Schleck CD, Ilstrup DM, Washington JA 2nd, Wilson WR. Optimal testing parameters for blood cultures. Clin Infect Dis. 2004 Jun 15;38(12):1724-30. Epub 2004 May 25.

15. Tarai B, Das P, Kumar D, Budhiraja S. Comparative evaluation of paired blood culture (aerobic/aerobic) and single blood culture, along with clinical importance in catheter versus peripheral line at a tertiary care hospital. Indian Journal of Medical Microbiology 2012; 30 (2) : 187 - 92. doi: 10. 4103 / 0255 - 0857. 96689.

Available online at: $\underline{\text { www.ijmrr.in }} 1248$ | P a g e 
16. Riley JA, Heiter BJ, Bourbeau PP. Comparison of recovery of blood culture isolates from two BacT/ALERT FAN aerobic blood culture bottles with recovery from one FAN aerobic bottle and one FAN anaerobic bottle. J Clin Microbiol.2003Jan;41(1):213-7.

17. Bannister ER, Woods GL. Evaluation of routine anaerobic blood cultures in the BacT/Alert blood culture system.Am JClinPathol.1995Sep;104(3):279-82.

18. Cornish N, Kirkley BA, Easley KA, Washington JA. Reassessment of the incubation time in a controlled clinical comparison of the BacT/Alert aerobic FAN bottle and standard anaerobic bottle used aerobically for the detection of bloodstream infections. Diagn Microbiol Infect Dis. 1998 Sept; 32(1):1-7. doi: 10.1016/50732-8893(98)00057-1.

19. Morris AJ, Wilson ML, Mirrett S, Reller LB. Rationale for selective use of anaerobic blood cultures. J Clin Microbiol. 1993 Aug;31(8):2110-3.

20. Sharp SE, McLaughlin JC, Goodman JM, Moore J, Spanos SM, Keller DW 3rd, Poppiti RJ Jr. Clinical assessment of anaerobic isolates from blood cultures. Diagn Microbiol Infect Dis. 1993 Jul;17(1):19-22.

21. Zenebe T, Kannan S, Yilma D, Beyene G. Invasive Bacterial Pathogens and their antibiotic susceptibility patterns in Jimma University specialized Hospital, Jimma, South West Ethiopia. Ethiop J Health Sci. 2011 Mar, 21(1);1-8.

22. Ali J, Kebede Y. Frequency of isolation and antimicrobial susceptibility pattern of bacterial isolates from blood culture, Gondar University teaching hospital, Northwest Ethiopia. Ethiop Med J. 2008 Apr;46(2):155-61.

23. Obi CL, Mazarura E. Aerobic bacteria isolated from blood cultures of patients and their antibiotic susceptibilities in Harare, Zimbabwe. Cent Afr J Med. 1996 Dec;42(12):332-6.
24. Shitaye D, Asrat D, Woldeamanuel Y, Worku B. Risk factors and etiology of neonatal sepsis in Tikur Anbessa University Hospital, Ethiopia. Ethiop Med J. 2010 Jan;48(1):11-21.

25. Nwadioha I, Nwokedi EOP, Kashibu E, Odimayo MS, Okwori EE. A review of bacterial isolates in blood cultures of children with suspected septicemia in a Nigerian. African J Microbiol Res. 2010 Feb,4(4);222-225.

26. Elbashier AM, Malik AG, Knot AP. Blood stream infections: micro-organisms, risk factors and mortality rate in Qatif Central Hospital. Ann Saudi Med. 1998 Mar-Apr;18(2):176-80.

27. Meremo A, Mshana SE, Kidenya BR, Kabangila R, Peck R, Kataraihya JB. High prevalence of Nontyphoid salmonella bacteraemia among febrile HIV adult patients admitted at a tertiary Hospital, NorthWestern Tanzania. Int Arch Med. 2012 Oct, 5(1) 28. Doi: 10.1186/1755-7682-5-28.

28. Asrat D, Amanuel YN. Prevalence and antibiotic susceptibility pattern of bacterial isolates from blood culture in Tikur Anbessa hospital, Addis Ababa. Ethiopia. Ethiop Med J. 2001 Apr,39(2);97-104.

29. Rina K, Nadeem SR, Kee PN, Parasakthi N. Etiology of blood culture isolates among patients in a multidisciplinary teaching hospital in Kuala Lumpur. J Microbiol Immunol Infect. 2007 Oct,40(5);432-437.

30. Rahbar M, Gra-Agaji R, Hashemi S. Nosocomial blood stream infections in Imam Khomeini Hospital, Urmia, Islamic Republic of Iran, 1999-2001. East Mediterr Health J. 2005 May;11(3):478-84.

31. Rahkonen M, Luttinen S, Koskela M, Hautala T. True bacteremias caused by coagulase negative Staphylococcus are difficult to distinguish from blood culture contaminants. Eur J Clin Microbiol Infect Dis. 2012 Mar, 31.

\section{How to cite this article?}

Karma G. Dolma, Deepan Gautam, Kh. Pooja Devi, T. S. K. Singh. A comparative study on the efficacy of first vs second blood culture set in the diagnosis of bacteraemia in Central Referral Hospital, Sikkim, India. Int J Med Res Rev 2016;4 (7):1243-1249.doi:10.17511/ijmrr.2016.i07.29. 